\title{
RETRACTED ARTICLE: Stress Response of Entomopathogenic Fungus Isaria fumosorosea to Copper
}

\section{Shaukat Ali • Zhen Huang • Shunxiang Ren}

Received: 29 November 2010 / Accepted: 21 December 2010 /

Published online: 11 January 2011

(C) Springer Science+Business Media, LLC 2011

This article has been retracted due to copyright issues that cannot be resolved.

This article has been retracted due to copyright issues that cannot be resolved.

Zhen Huang is joint first author

S. Ali $\cdot$ Z. Huang $\cdot$ S. Ren $(\bowtie)$

Engineering Research Center of Biological Control, Ministry of Education, College of Natural Resource and Environment, South China Agricultural University, Wushan Road, 510642 Guangzhou City, People's Republic of China

e-mail: rensxcn@yahoo.com.cn

S. Ali

e-mail: alientomologist@gmail.com

Z. Huang

e-mail: hzscau@scau.edu.cn 